\title{
Effect of Bisphenol A on the Testis of Offspring of White Albino Rats during Pregnancy and Lactation: (Light and Electron Microscopic Study)
} Al Sayed A. Abd Elhady*1, Mohamed A. Autifi², Moustafa E. El Gizawy ${ }^{2}$, Moaaz M. Yousef ${ }^{2}$ ${ }^{1}$ Department of Human Anatomy and Embryology, Faculty of Medicine, Helwan University, Egypt.

${ }^{2}$ Department of Human Anatomy and Embryology, Faculty of Medicine, Al-Azhar University, Egypt. *Corresponding author: Al Sayed A. Abd Elhady, Mobile: (+20) 01001951390, E-Mail: abdrahmankellany @ yahoo.com

\begin{abstract}
Introduction: The synthetic industrial and biomedical chemicals suspected to threat the health of the living organisms by disrupting their endocrine systems, referred to as endocrine disrupting chemicals (EDCs).

Objective: To study the effects of Bisphenol A (BPA) on the natal and postnatal testis by the light and electron microscope to make sure if it is harmful or not on the development of male reproductive organs in albino rats.

Matrial and Methods: Chemical used: Bisphenol A. The daily single oral dose was $(\mathbf{0 . 3} \mathbf{~ m g} / \mathbf{k g ~ B W . / d a y})$. Experimental animals: Adult female rats in proestrus cycle were caged overnight with adult males of proven fertility ( 1 male / 2 females / cage) and produced neonatal male rats.The offspring rats were divided into five main groups: Group I (Control offspring group), Group II (Experimental low dose treated offspring group with Bisphenol A $(\mathbf{0 . 3} \mathbf{~ m g} / \mathbf{k g ~ B W . / d a y ) ~ f o r ~ t w o ~ w e e k s , ~ G r o u p ~ I I I ~ ( E x p e r i m e n t a l ~ h i g h ~ d o s e ~ t r e a t e d ~ o f f s p r i n g ~ g r o u p ; ~} 12 \mathrm{mg} / \mathrm{kg}$ b.w/day), Group IV (First recovery group ), Group V (Second recovery group),

Results: Chronic Bisphenol A administration produced testicular toxicity leading to marked serious histological changes of the testis including the spermatogenic cells, spermatids, Sertoli cells and Leydig cells. These testicular changes increased with the high dose intake of BPA indicating its cumulative toxic effects that caused hypospermatogenesis, leading to infertility.

Conclusion: Bisphenol A administration causes testicular toxicity. So, these serious complications on testicular structure should be considered when buying any canned foods or drinks, because it is used nowadays on a large scale allover the world.
\end{abstract}

Keywards: Albino Rats, BPA, Testis. Histology.

\section{INTRODUCTION}

The Endocrine Society defines an endocrinedisrupting chemical (EDC) as "an exogenous chemical, or mixture of chemicals, that interferes with any aspect of hormone action" (1). Bisphenol A (BPA) is an EDCs that is widely present in the environment. It is widely used in production of polycarbonate plastics which are introduced into the environment by the pharmaceutical, food, cosmetics, agricultural, and chemical industries (2). A key concept in the study of EDCs is that their mechanism of action cannot be easily demonstrated in vivo, as their effects can appear after prolonged/continuous exposure to a low dose and, even worse, these effects are often the result of the simultaneous interaction of several substances (mixture effect) and the hormone balance of the individual concerned $^{(\mathbf{3})}$. Spermatogenesis is a complex differentiation process involving the production of spermatozoa which requires the functional coordination of numerous endocrine and paracrine factors. For this reason, EDCs have been repeatedly linked to its disruption ${ }^{(4)}$.

Several researches on EDCs have shown that their effects in critical periods of development, including prenatal life and early childhood, may lead to serious consequences. However, the studies that examine the effects of prenatal and lactational exposures are extremely limited ${ }^{(5)}$. Some studies suggested that BPA affects male reproductive system, leading to lower sperm counts and alterations in testicular histology ${ }^{(6)}$.

But, the effects and mechanism of BPA toxicity on reproductive system still remain unclear. So, the majority of population in the developing countries may not be aware of the harmful effects of BPA on the human body ${ }^{(7)}$. Hence, this study was designed to observe and analyze the effects of prenatal and post natal exposure of BPA on the testes of adult male albino rats and the possibility of recovery.

\section{MATRIALS AND METHODS \\ A-Materials: \\ 1- Chemical used:}

Bisphenol A (BPA, CAS 80-05-7, > 99\% pure) was purchased from Sigma Aldrich Company (USA) Tocopherol-stripped corn oil (ICN Biomedicals Inc., Aurora, $\mathrm{OH}$ ) served as the vehicle and control substance. The daily single oral dose was $0.3 \mathrm{mg} / \mathrm{kg}$ b.w/day. while the high dose was $12 \mathrm{mg} / \mathrm{kg}$ b.w/day. The doses were selected as lowest observed adverse effect levels (LOAEL) on the male reproductive system and toxic daily doses of BPA according to $(8,9)$.

\section{2- Experimental animals:}

Adult male and female albino rats (weight 200-250 gm male and 160-200gm female ) were selected in Animal 
House of Veterinary Serum \& Vaccine Research Institute (Abbasya,Cairo,Egypt). They were maintained in light and dark cycles of 12 hours each, in a controlled environment and fed with standard food diet and water. Adult female rats in proestrus cycle were caged overnight with adult males of proven fertility (1 male / 2 females / cage). Females were examined daily using vaginal smear technique to ensure that they were in regular estrous cycle, The day on which the sperm was found in the vagina was designated as day 1 of gestation (GD). Each pregnant female was isolated in a cage to stay until the day of birth (PND 0). The pregnant females are weighted and received a tolerant dose of BPA ( 0.3 or $12 \mathrm{mg} / \mathrm{kg} \mathrm{BW} . /$ day) (according to regimen used) dissolved in corn oil via gavage daily from day six of pregnancy, through gestation, during lactation till weaning of their offspring (21 days old). The study was approved by Ethical Committee of Faculty of Medicine, Al-azhar University, Cairo.

\section{Animal grouping: \\ The animals were divided into five main groups: \\ 1) Control offspring group (G. 1): 10 rats}

The pregnant females received equivalent dose of corn oil by gavage daily from day six of pregnancy, through gestation, during lactation till weaning of their offspring (21 days old). The weaned rat offspring of control group (10 males) are still administered postnatally daily the same equivalent dose of corn oil by gavage that given for their mothers till sacrification (35 days old ).

2) Experimental low dose treated offspring group (G. 2): 10 rats

The pregnant females are weighted and received a tolerant dose of BPA (low dose treatment) $(0.3 \mathrm{mg} / \mathrm{kg}$ BW./day) dissolved in corn oil via gavage daily from day six of pregnancy, through gestation, during lactation till weaning of their offspring ( 21 days old). The weaned rats of this group (10 males) are weighted and received daily doses of their equivalent from the same doses that given for their mothers by gavage till sacrification (35 days old).

\section{3) Experimental high dose treated offspring group} (G. 3): 10 rats

The pregnant females are weighted and dosed a high dose of BPA (12 mg/kg BW. /day) daily dissolved in corn oil via gavage from Day six of pregnancy, through gestation, during lactation till weaning of their offsprings ( 21 days old). The weaned rats of this group (10 males) are weighted and received daily doses of their equivalent from the same doses that given for their mothers till sacrification (35 days old).

\section{4) First recovery group (G. 4): 10 rats}

The pregnant females are weighted and received a tolerant dose of BPA (low dose treatment) $(0.3 \mathrm{mg} / \mathrm{kg}$ BW/day) dissolved in corn oil via gavage from day six of pregnancy, through gestation, during lactation till weaning of their offspring ( 21 days old), The weaned rats of this group (10 males) are weighted and administered daily the same equivalent dose of corn oil by gavage that given for the control offspring rats till sacrification ( 35 days old ).

5) Second recovery group (G.5): 10 rats

The pregnant females are weighted and dosed a high dose of BPA (12 mg/kg BW/day) daily dissolved in corn oil via gavage as Toxic Daily Intake dose for human according to Vom and Hughes ${ }^{(8)}$ from Day six of pregnancy, through gestation, during lactation till weaning of their offsprings (21 days old), The weaned rats of this group (10 males) are weighted and administered daily the same equivalent dose of corn oil by gavage that given for the control offspring rats till sacrification (35 days old ).

\section{B- Methods:}

\section{I- Histological study:}

The left testis was divided into two pieces, they first one was fixed in Bouin's fixation solution for $18 \mathrm{~h}$, and tissue samples were processed, Slides were stained with Hematoxylin \& Eosin and Masson's trichrome stain and examined using a light microscope. The second piece were fixed with glutraldehyde, processed and cut with glass knives for ultrathin sections (60-70 $\mathrm{nm}$ ) which mounted on copper grids. The ultra thin section poststained with uranyl acetate and lead citrate and examined by a Joel TEM CS 100 at the Regional Center for Mycology And Biotechnology, Al Azhar University,Cairo.

\section{II-Statistical analysis}

The Statistical data included were expressed as mean, standard error (SE) standard deviation(SD \pm ) and the student $\mathrm{T}$ test was used to elucidate the differences between the treated groups, recovery groups and control group. The obtained data were analysed by using SPSS software (V. 21).

\section{RESULTS}

\section{I- Group I (Control Group):}

A) Light microscopic results:

1) Hematoxylin and Eosin (Fig. 1): The testis revealed the normal structure, showing seminiferous tubules surrounded by basal lamina and peritubular myoid cells and contain Sertoli cells, spermatogonia type A and primary spermatocytes and immature rounded spermatids. Seminiferous tubules appear closely packed with the interstitial tissue in between the empty lumen of tubules containing the cell of Leydig.

2) Masson's trichrome stained sections (Fig.6).

Minimal deposition of collagen fibers is seen in the basement membranes surrounding the tubules and in the interstitial tissues.

\section{B) Electron microscopic results (Figs. 11-15):}

Examination of the sections showing the seminiferous tubules were lined by spermatogonia, primary spermatocytes, early round spermatids and Sertoli cells, the tubules were surrounded by myoid 
cells with flat nuclei in their basal laminae. Sertoli cells were located within the spermatogenic epithelium and characterized by its indented nuclei and prominent nucleolus, Spermatogonia appear with ovoid nuclei and peripheral marginated heterochromatin. The primary spermatocytes have large rounded nuclei with clumps of heterochromatin. The early spermatid possessed a spherical euchromatic nucleus with prominent nucleolus, acrosomal cap, their cytoplasm showed peripherally arranged mitochondria.

\section{II- Results of the experimental treated group}

(G. 2):

A) Light microscopic results:

1) Hematoxyline \& Eosin (Fig.2)

- The majority of the tubules exhibited a normal histological appearance, but some of them showed structural variations, mild loss in the spermatogonia and the spermatocytes suggesting that the process of degeneration starts at foci within each tubule, minimal spaces between the cells appeared in some tubules.

- Spermatogonia showed small pyknotic nucleus rested on arelatively thick and an irregular B.M, while the primary spermatocyte showed condensed chromatin in its nucleus.

- Considerable variations from apparently normal tubules to irregularly damaged ones were detected in this group, such damaged tubules were randomly distributed and adjoined the normal ones, a slight hypospermatogenesis was manifested by a mild diminution in the thickness of seminiferous epithelium and in the germ cell number.

- The atrophied tubules have small and few vacuolar spaces replaced the lost cells, the spaces formed a small outward projection pushing the basement membrane together with the outer layer of spermatogenic epithelium.

- The interstitium also, showed dilated congested blood vessels and vacuoles.

\section{2) Masson's trichrome stained sections (Fig.7):}

Moderate collagen deposition is seen in the basement membranes surrounding the atrophied tubules, and in the interstitial tissues.

B) Ultrastructuraly, sections (Figs 16\&17):

- Most of the primary spermatocytes are normal, some exhibit nuclei showed irregular nuclear envelope or shrunked nucleus, furthermore, their cytoplasm showed few small vacuoles.

- Most of Sertoli cells are normal, few with irregular nuclei which became irregular in shape and contained irregular nuclear envelope and peripherally marginated chromatin, their cytoplasm showed small few vacuoles.
III- Results of the experimental treated group (G. 3):

A) Light microscopic results:

1) Hematoxyline $\&$ Eosin stained sections (Fig.3):

- The majority of the tubules exhibited abnormal histological appearance, some tubules revealed absence of a part of their tubular wall, the lost cells were mostly the spermatogonia and the spermatocytes suggesting that the process of degeneration starts at foci within each tubule.

- A maturational arrest with clear diminution in the thickness of the epithelium, a low number of spermatogonia, spermatocytes and spermatids, the majority of tubules were full regressed at times normal tubules undergoing complete spermatogenesis appeared directly adjacent to regressed tubules, the regressed tubules showed more thickened and wrinkled basement membrane and contained few germ cells.

- The atrophied tubules have multiple large vacuolar space replaced the lost cells, such spaces formed a small outward projection pushing the basement membrane together with the outer layer of spermatogenic epithelium.

2) Masson's trichrome stained sections (Fig.8) : Marked collagen deposition in the the basement membranes surrounding the atrophied tubules.

B) Ultrastructuraly, sections (Figs.18-21):

- Most of the spermatogonia with irregular outlined nucleus and condensed peripheral heterochromatin, shrunked nucleus, the cell is seen lying on an irregular thick basement membrane with myoid cell, its cytoplasm shows distorted mitochondria and electron dense, Presence of multiple wide intercellular spaces and intracellular vacuoles are also seen.

- Most of the nuclei of sertoli cells became irregular in shape and contained irregular nuclear envelope, their cytoplasm showed multiple vacuoles.

- Most of the primary spermatocytes exhibited nuclei containing clumps of heterochromatin, furthermore, there cytoplasm showed vacuoles, the mitochondria were distorted.

- Most of the spermatids showed nuclei with degenerative changes such as irregular or shrunken nuclear envelope (apoptotic nucleus) with complete or partial loss of acrosomal cap, other showed ruptured nucleus, the cytoplasm of the other spermatid contains few and distorted mitochondria, multiple wide vacuolated intercellular spaces.

- Most of the interstitial Leydig cell show shrunked nucleus.

V-Results of the first recovery group (G.4):

A) Light microscopic results:

1) Hematoxyline \& Eosin stained sections (Fig.4):

There was a recovery in lumen size and restoration of seminiferous tubule structure with 
increase in germ cell: spermatogonia, spermatocytes, spermatids and spermtozoa as compared to treated group. There was a decline in damage induced by BPA reduction in vacuolization, pyknosis and intact structure of germinal epithelium. There was a significant restoration in the germ cells towards the peripheral region of seminiferous tubules. There was significant reduction in the abnormalities as compared to the BPA induced tissue. There was a prominent recovery in the size of seminiferous tubules and lumen was filled with spermatozoa.

2) Masson's trichrome stained sections (Fig.9): showed minimal deposition of collagen fibers is seen in basement membranes of the tubules and in the interstitium around seminiferous tubules.

B) Ultrastructuraly, sections (Figs 22 \& 23):

Spermatogonia A with condensed chromatin in their nuclei resting on basement membrane, their cytoplasm contain mitochondria with disturped cristae. Primary spermatocyte show som vacuoles and few organell while sertoli cell show indented nucleus with aprominent nucleolus.

spermatids with large ovoid euchromatic nuclei. Their cytoplasm contains peripherally located mitochondria. The cytoplasmic processes of sertoli cells were seen in between the spermatids.

\section{V-Results of the second recovery group (G.5):}

A) Light microscopic results

1) Hematoxyline \& Eosin stained (Fig.5):
There was a mild recovery and restoration of seminiferous tubule structure with mild increase in germ cell: spermatogonia, spermatocytes, spermatids and spermtozoa as compared to group (4). There was a moderate damage induced by BPA as vacuolization, pyknosis. There was a mild restoration in the germ cells towards the peripheral region of seminiferous tubules There was mild reduction in the abnormalities as compared to the group (4). There was a mild recovery in the size of seminiferous tubules and lumen contains few spermatozoa.

2) Masson's trichromestained sections, (Fig.10):

Showed amarked collagen deposition in the capsule and amoderate deposition in the basement membranes.

B) Ultrastructuraly, sections (Fig. 24):

Spermatogonia, primary spermatocytes with rounded nuclei. Their cytoplasm contains few multiple vacuoles, Sertoli cells appear with indented nucleus, prominent nucleolus with multiple mitochondrial vacuoles, irregular thickened basement membrane.

\section{Statistical results:}

A significant increase $(\mathrm{P}<0.05)$ in deposition of collagen fibers is seen in the basement membranes of the tubules and in the interstitium around seminiferous tubules of all treated and recovery groups in comparison to those in group I control rats (Table 1 \& Fig. 23).

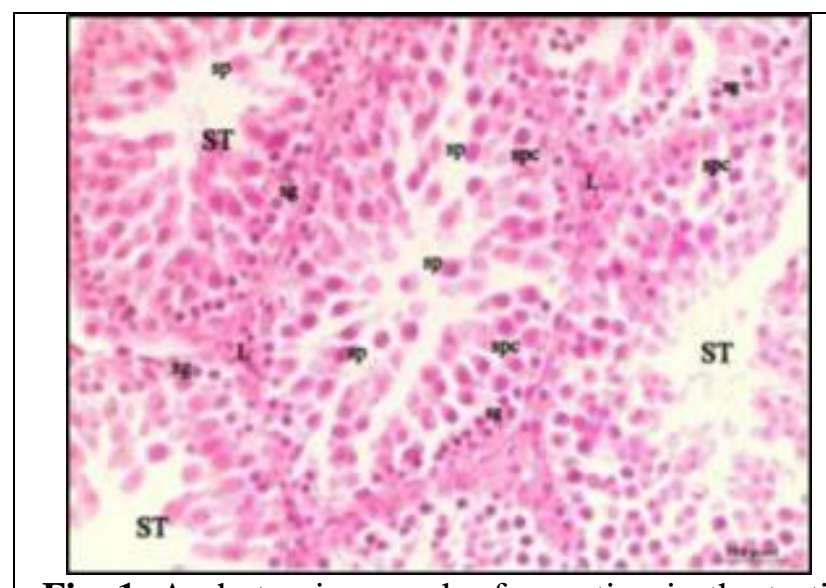

Fig. 1: A photomicrograph of a section in the testis of control group (G.1) showing seminiferous tubules (ST) contain spermatogonia type A (sg) and primary spermatocytes $(\mathrm{SPc})$ and immature round spermatid (SP), no sperms are present. Seminiferous tubules appear closely packed with the interstitial tissue containing leydig cells (L) (H. \& E. X 400).

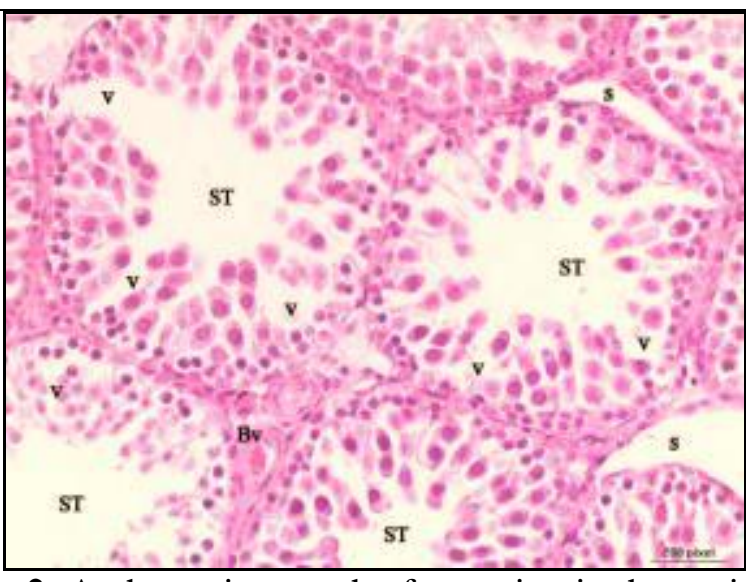

Fig. 2: A photomicrograph of a section in the testis of treated group (G.2), showing mild degeneration of seminiferous tubules (ST) in the form of mild sloughing of germ cell $(\mathrm{S})$ from the basement membrane, some germ cells are replaced by vacuolation spaces (V) in the epithelium. The interstitium showed congested blood vessels (BV). (H. \& E X400). 


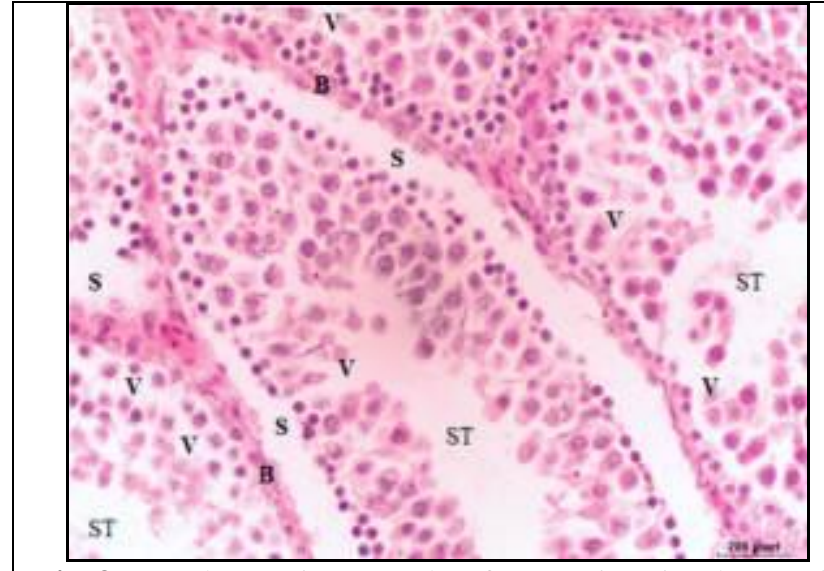

Fig.3: A photomicrograph of a section in the testis of high dose treated group (G.3), showing a degenerated seminiferous tubules (ST) with marked epithelial sloughing (S) from the thickened basement membrane (B) and marked loss of germ cells replaced by spaces in the epithelium $(\mathrm{V})$ and absence of immature round spermatid (H. \& E. $\mathrm{X400).}$

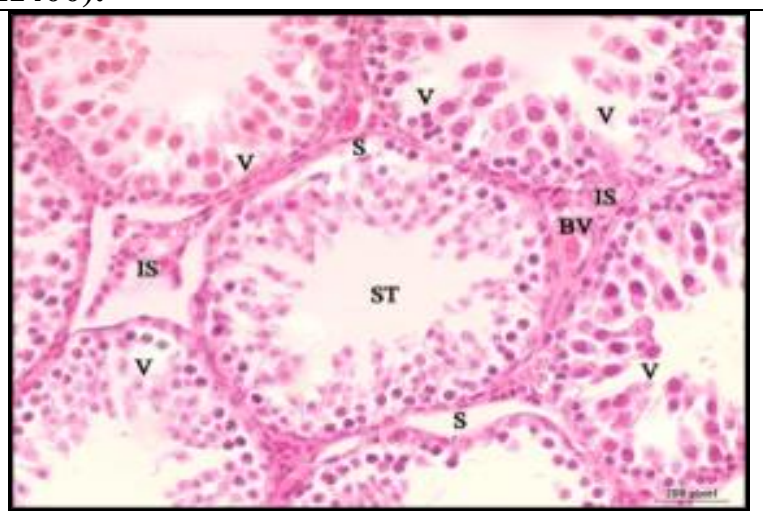

Fig. 5: A photomicrograph of a section in the testis of the second recovery group (G.5) showing improvement and a restoration of the seminiferous tubule structure (ST), mild sloughing of the germinal cells from the basement membrane (S) some lost germ cells are replaced by spaces $(\mathrm{V})$ in the epithelium. The interstitium showed congested blood vessels (BV). (H. \& E X400).

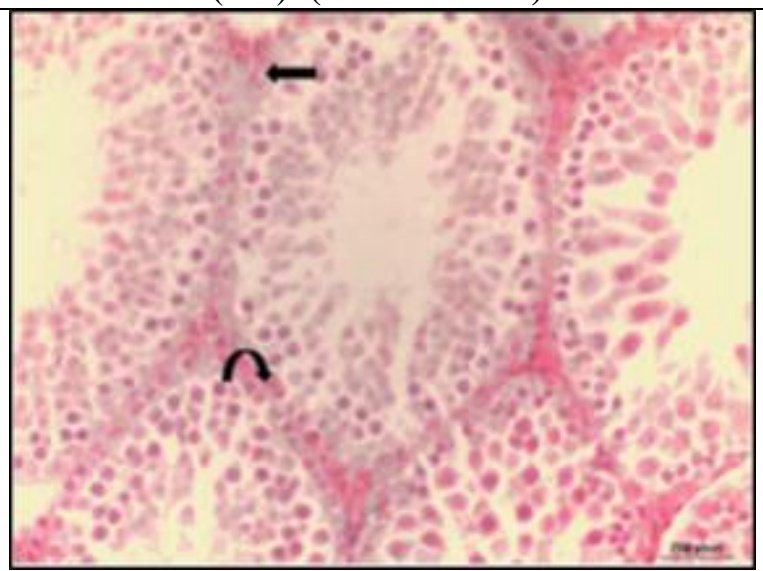

Fig.7: A photomicrograph of a section in the testis of the treated group (G.2) showing moderate collagen deposition in the basement membranes (curved arrow) and interstitium (straight black arrow) (Masson's trichrome X400).

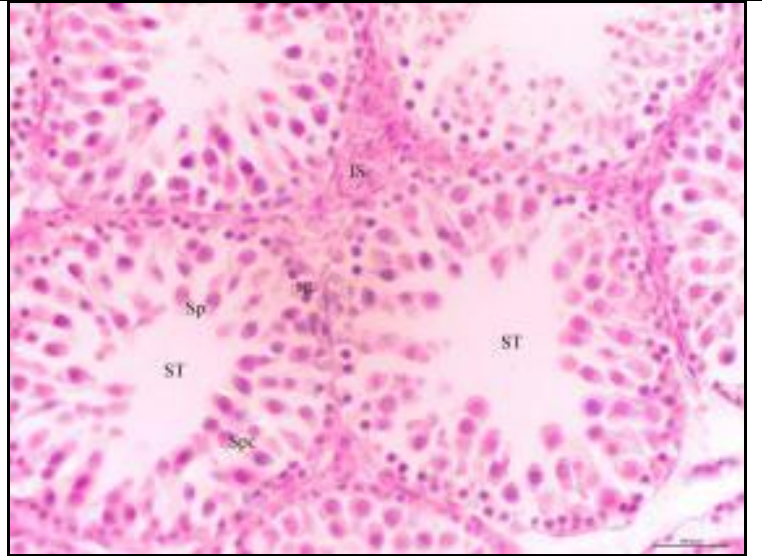

Fig.4: A photomicrograph of a section in the testis of the first recovery group (G.4) seminiferous tubules showing a restoration of seminiferous tubule structure with an increase in germ cell number, spermatogonia type A (sg) and primary spermatocytes (SPc) and immature round spermatid (SP)with no congestion in the interstitial space (IS) (Hx. \& E. X400).

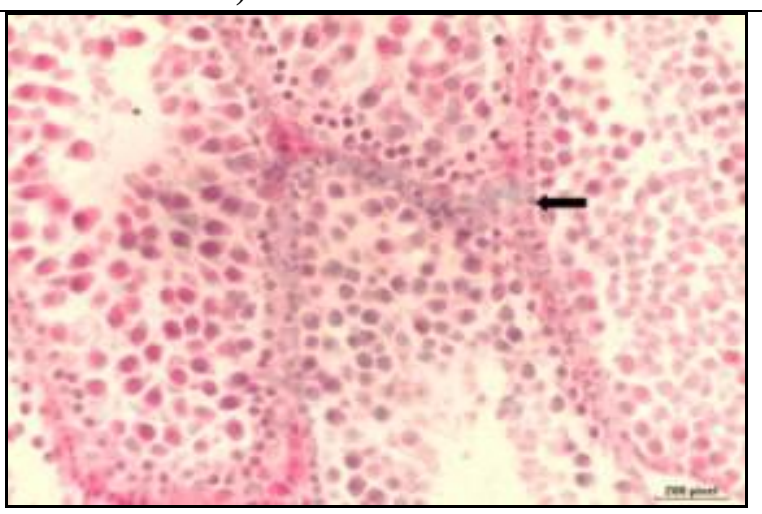

Fig. 6: A photomicrograph of a section in the testis of control group (G.1) showing minimal deposition of collagen fibers is seen in basement membranes of the tubules and in the interstitium around the seminiferous tubules(black arrow) (Masson's trichrome X400).

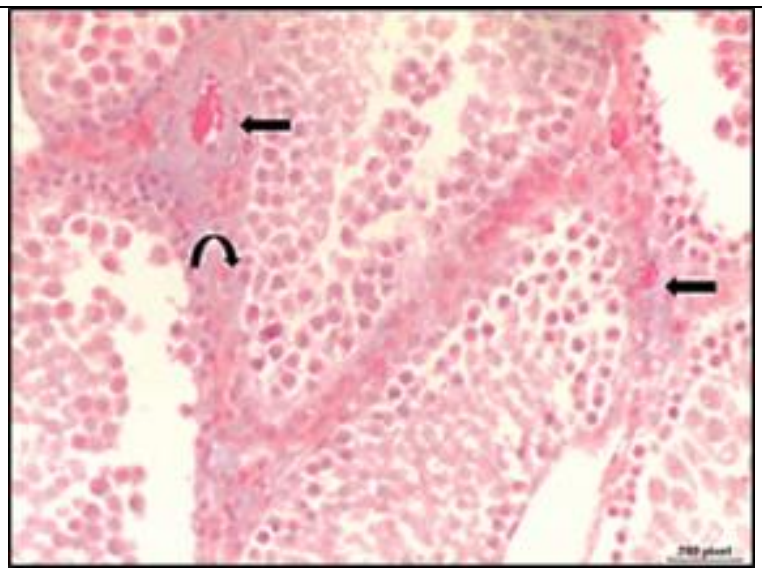

Fig. 8: A photomicrograph of a section in the testis of the high dose treated group showing a marked collagen deposition in the the basement membranes (curved arrow) and around the congested blood vessels in the interstitial tissue (straight black arrows) (Masson's trichrome X400). 


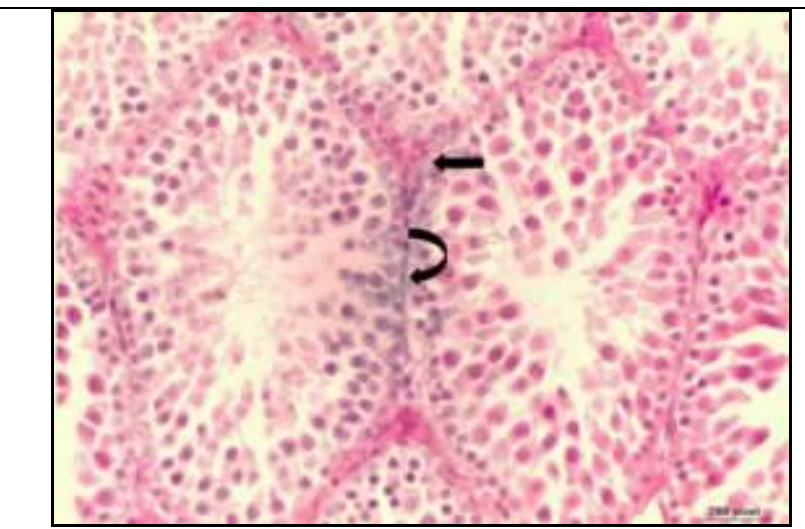

Fig.9: A photomicrograph of a section in the testis of the first recovery group (G.4) showing a minimal deposition of collagen fibers is seen in basement membranes of the tubules (curved arrow) and in the interstitium around seminiferous tubules (straight black arrow) (Masson's trichrome X400).

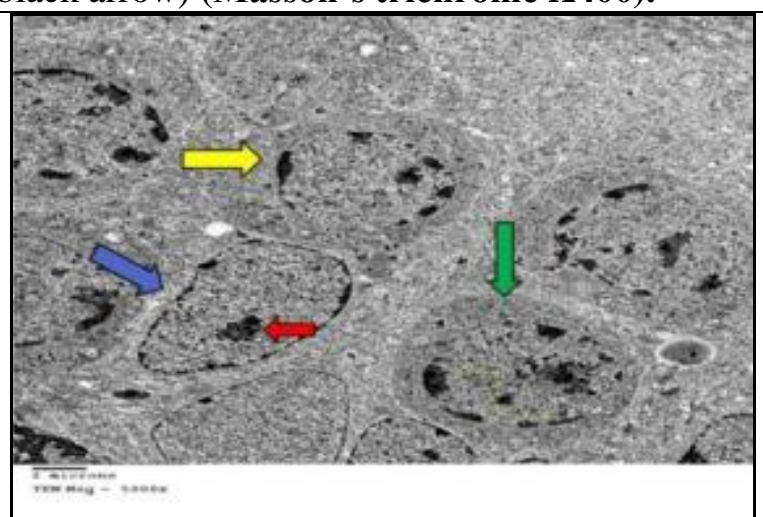

Fig. 11: An electron photomicrograph of an ultrathin section in the testis of control group (G.1) showing Sertoli cell with euochromatic nucleus (blue arrow) and prominent nucleolus (red arrow). Spermatogonia type A, with ovoid nucleus and peripheral marginated heterochromatin (green arrow). Primary spermatocyte is also seen with large rounded nucleus with clump of hetero chromatin (yellow arrow)

\section{(Uranyl acetate \& lead citrate X5000).}

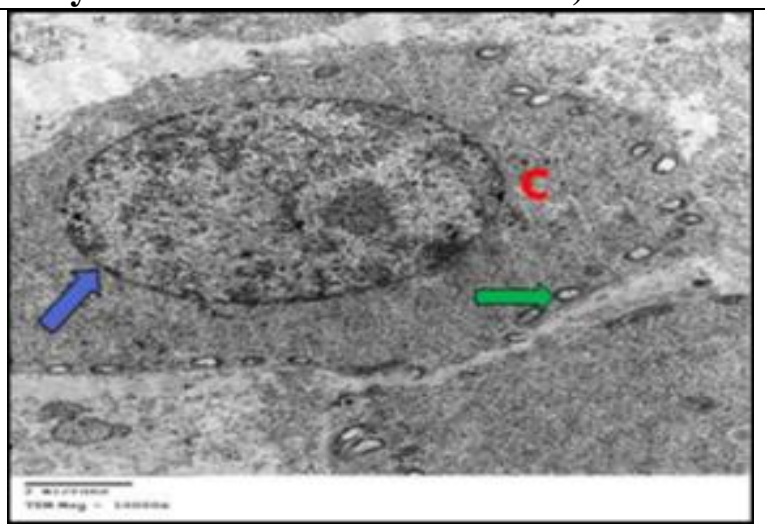

Fig. 13: An electron photomicrograph of an ultrathin section in the testis of control group (G.1) showing the normal round spermatid with round euchromatic nucleus (blue arrow) and prominent acrosomal cap (c). Their cytoplasm showed peripherally arranged mitochondria (green arrow) (Uranyl acetate\& lead citrate $\mathbf{X 1 0 0 0 0}$ ).

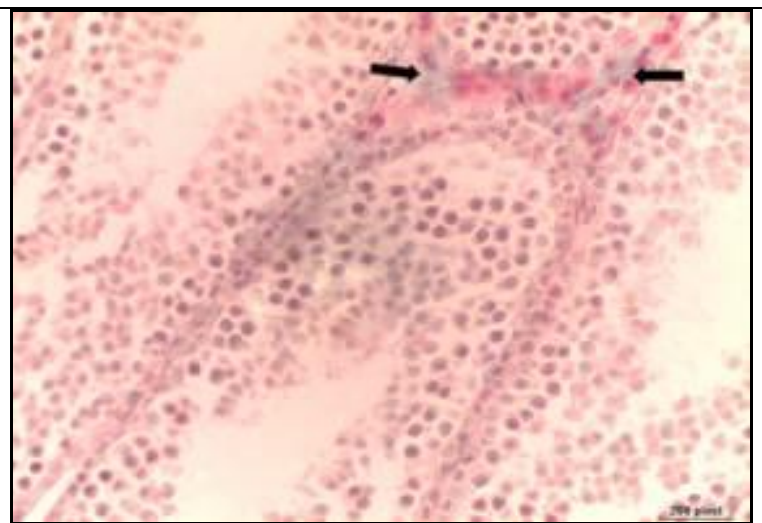

Fig. 10: A photomicrograph of a section in the testis of the second recovery group (G.5) showing moderate deposition in the basement membranes \& interstitial tissues (black arrows) (Masson's trichrome X400).

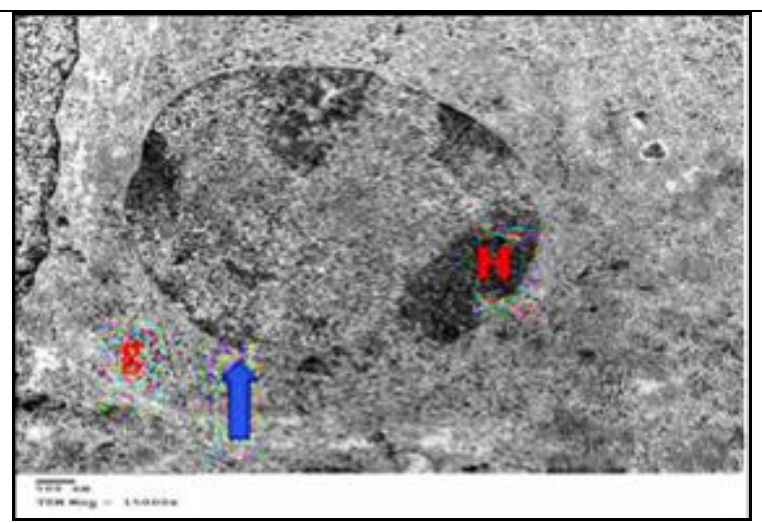

Fig. 12: An electron photomicrograph of an ultrathin section in the testis of control group (G.1) showing spermatogonia type A (g) with round nucleus (blue arrow) and peripherally marginated heterochromatin (H) (Uranyl acetate\& lead citrate X15000).

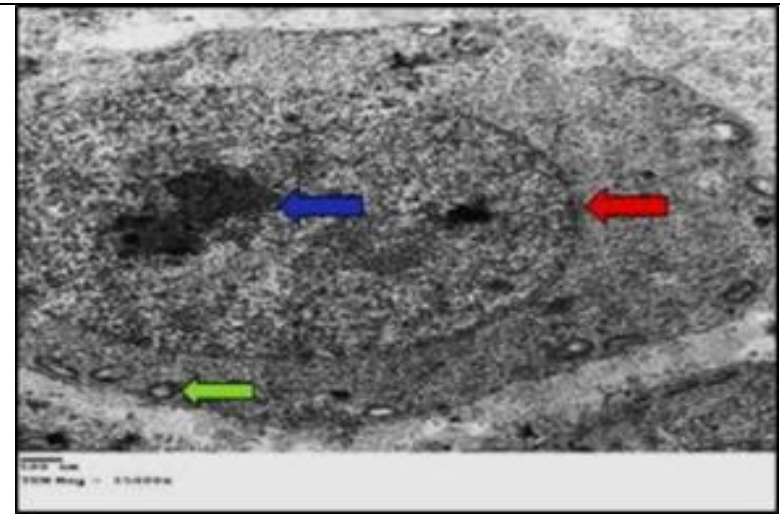

Fig.14: An electron photomicrograph of an ultrathin section in the testis of control group (G.1) showing the normal round spermatid with round euchromatic nucleus and prominent acrosomal cap (red arrow) and prominent nucleolus (blue arrow).Their cytoplasm showed peripherally arranged mitochondria (green arrow) (Uranyl acetate\& lead citrate X15000). 


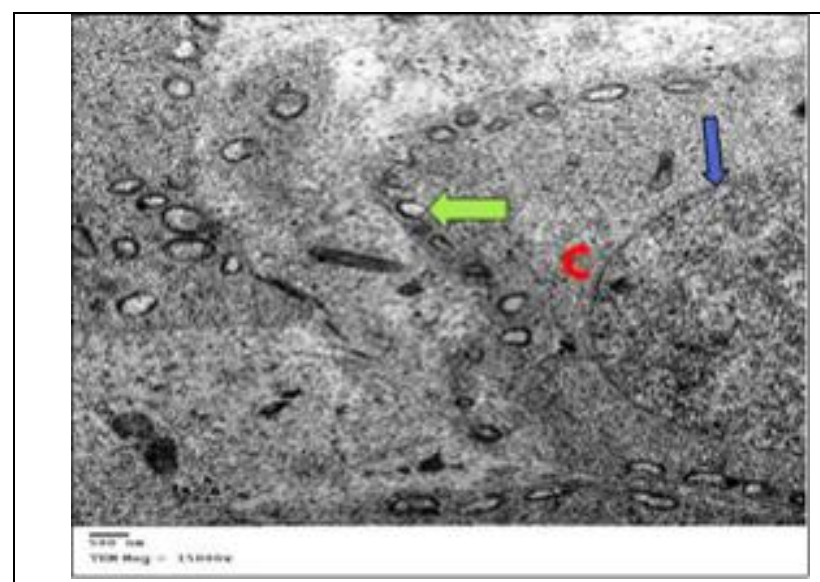

Fig. 15: An electron photomicrograph of an ultrathin section in the testis of control group (G.1) showing the normal round spermatid with round euchromatic nucleus and prominent acrosomal cap (C) and prominent nucleolus (blue arrow). Their cytoplasm showed peripherally arranged mitochondria (green arrow) (Uranyl acetate\& lead citrate X15000).

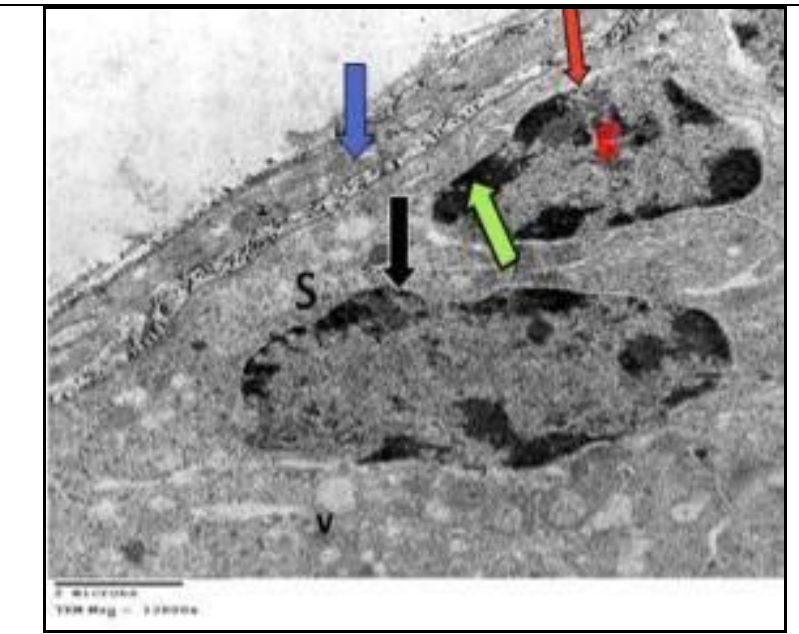

Fig.17: An electron photomicrograph of an ultrathin section in the testis of treated group (G.2), showing the spermatogonia $\mathrm{A}(\mathrm{g})$ resting on moderately thick and an irregular basement membrane (blue arrow). Spermatogonia A with irregular nuclear envelope (red arrow) and peripherally marginated heterochromatin (green arrow). Sertoli cell (S) with an ovoid nucleus with an irregullar nuclear envelope and a peripherally marginated heterochromatin (black arrow), their cytoplasm show small intra cellular vacuoles (v). (Uranyl acetate\& lead citrate X12000).

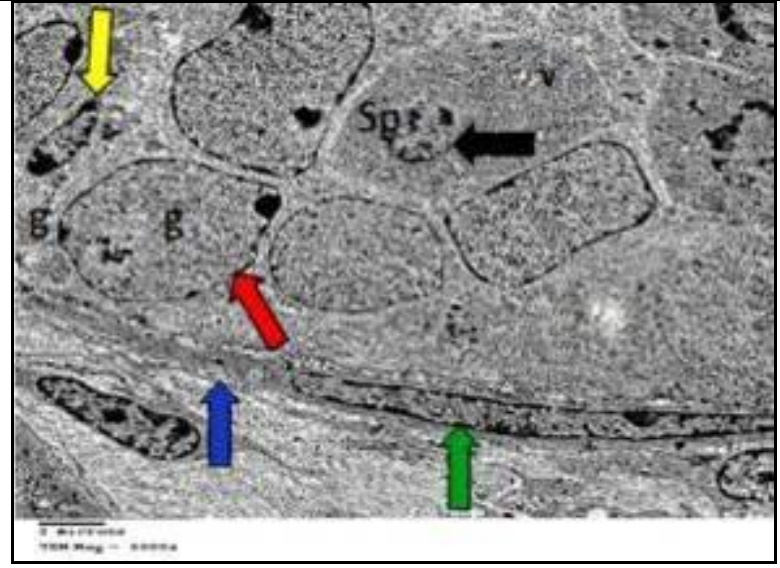

Fig. 16: An electron photomicrograph of an ultrathin section in the testis of low dose treated group (G.2), showing spermatogonia A (g) resting on amoderately thick and an irregular basement membrane (blue arrow) with myoid cell (green arrow), Spermatogonia A with an irregular nuclear envelope (red arrow) and other cell with an irregular a shrunken nucleus (yellow arrow). Primary spermatocyte (Sp) with shrunken euchromatic nucleus (black arrow) (Uranyl acetate\& lead citrate $\mathrm{X6000}$ ).

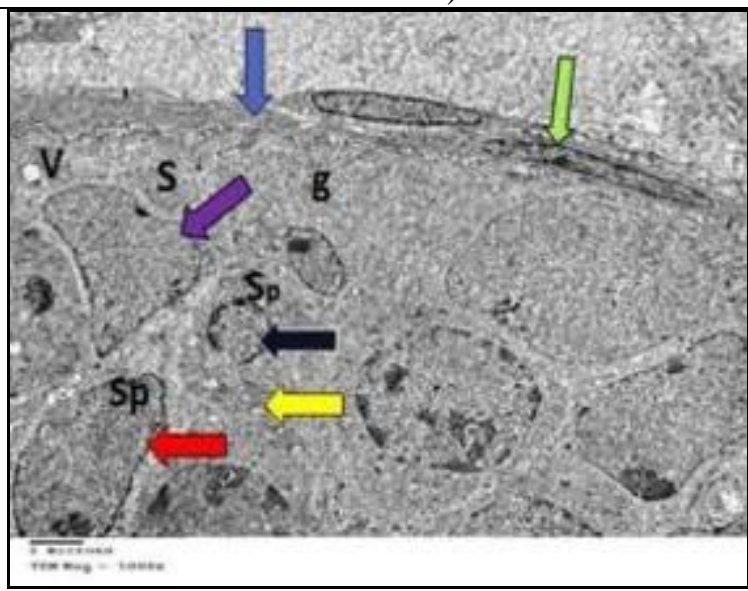

Fig.18: An electron micrograph of an ultrathin section in the testis of treated group (G3), showing spermatogonia A $(\mathrm{g})$ resting on very thick and irregular basement membrane (blue arrow) with myoid cell (green arrow). Spermatogonia A (g). Primary spermatocytes (sp), one cell with a shrunked nucleus (black arrow) and another cell with an irregular nuclear envolpe and peripherally marginated heterochromatin (red arrow), Their cytoplasm show distorted mitochondria (yellow arrow). Sertoli cell (S) with irregular nuclear envelope (violet arrow) large intracellular vacoules (v) (Uranyl acetate\& lead citrate X5000). 


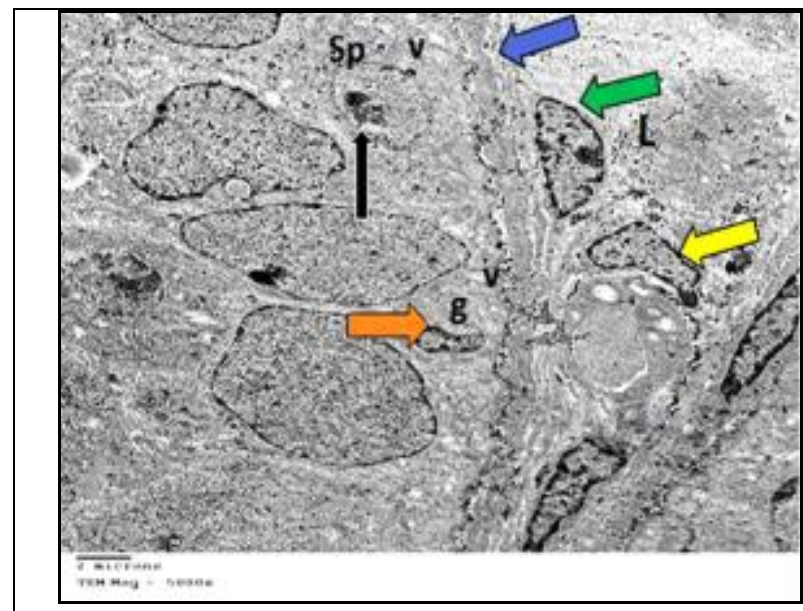

Fig. 19: An electron photomicrograph of an ultrathin section in the testis of treated group (G.3) showing spermatogonia A (g) resting on very thick and irregular basement membrane (blue arrow) ensheathed by a peritubular myoid cell (green arrow). spermatogonia with a shrunked nucleus (orange arrow), their cytoplasm showed large intracellular vacoules (v), cells are seen disrupted from basement membrane. Primary spermatocytes (Sp) with irregular nuclear envelope (black arrow) and peripherally marginated heterochromatin, their cytoplasm showed large intracellular vacoules (v). The interstitium showed leydig cells with a shrunked nucleus (yellow arrow) (Uranyl acetate\& lead citrate $\mathrm{X5000}$ ).

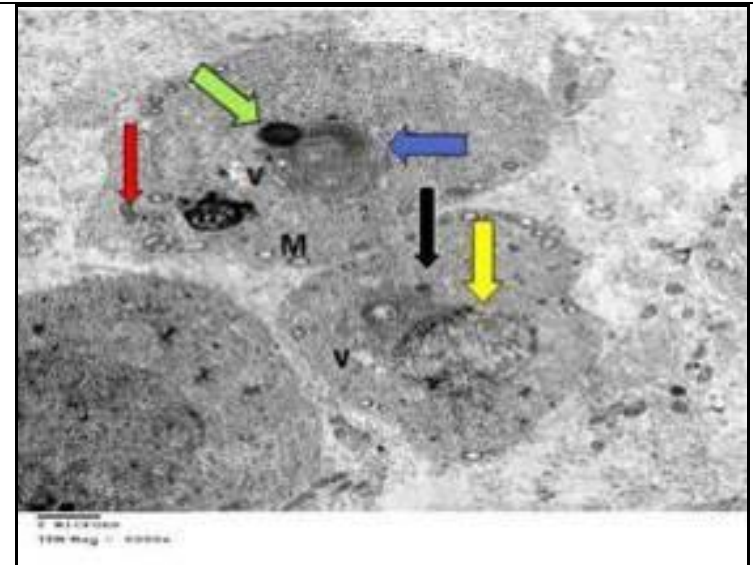

Fig. 21: An electron photomicrograph of an ultrathin section in the testis of G.3 showing a round spermatid with shrunked nucleus (blue arrow), an eccentric acrosomal cap (green arrow), its cytoplasm showed peripherally arranged mitochondria (M) some of them are distorted (red arrow) and intracellular vacuoles (v). Another spermatid with prominent mitochondria (black arrow) and an euchromatic nucleus (yellow arrows) (Uranyl acetate\& lead citrate x6000).

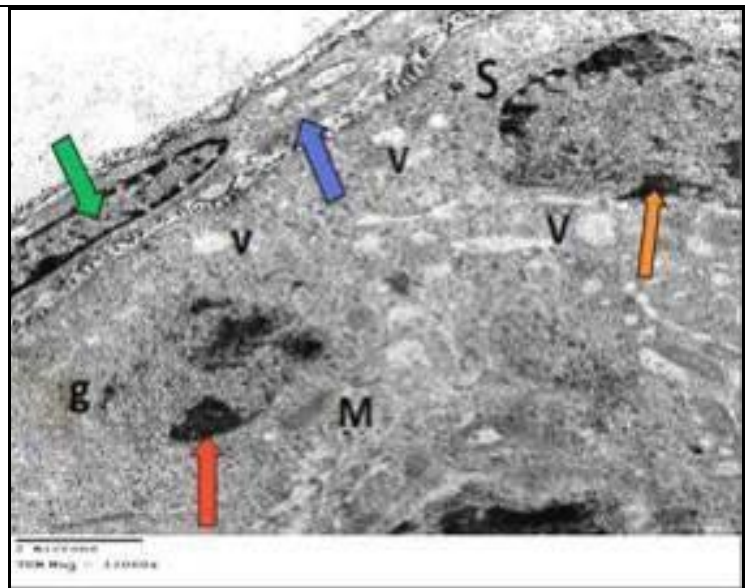

Fig. 20: An electron photomicrograph of an ultrathin section in the testis of treated group (G3) showing spermatogonia $(\mathrm{g})$ with irregular nuclear envelope and a peripherally marginated heterochromatin (red arrow). Their cytoplasm show large intracellular vacoules (v) and mitochondria (M). Resting on an irregular basement membrane (blue arrow) with myoid cell (green arrow). Sertoli cell (S) with an irregullar nuclear envelope and a peripherally marginated heterochromatin (orange arrow), their cytoplasm show large intercellular vacuoles (V) (Uranyl acetate\& lead citrate X12000).

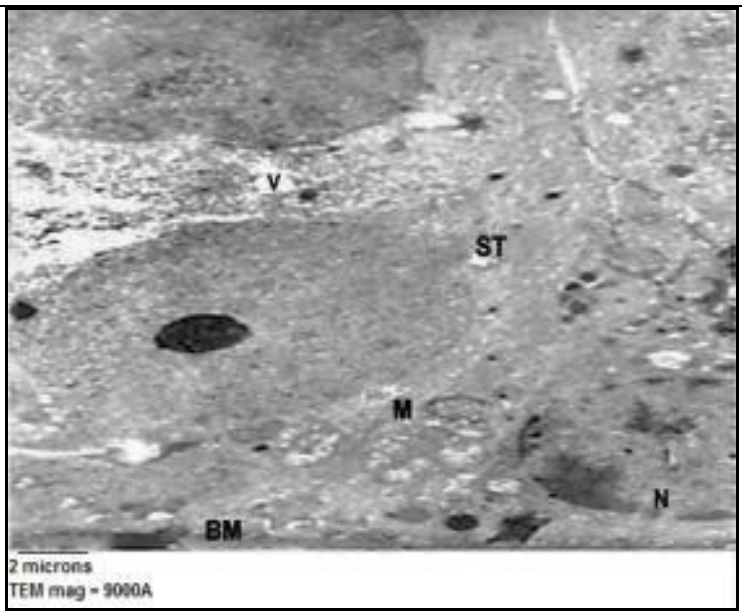

Fig. 22: An electron micrograph of an ultrathin section in the testis of the first recovery group (G4) showing spermatogonia A (g) wihh condensed chromatin in their nuclei $(\mathrm{N})$ resting on a basement membrane (BM), their cytoplasm contain mitochondria (M) with disturped cristae. Primary spermatocyte show som vacuoles $(\mathrm{V})$ and few organell while sertoli cell $(\mathrm{St})$ show an indented nucleus with a prominent nucleolus.(Uranyl acetate\& lead citrate X 9000) 


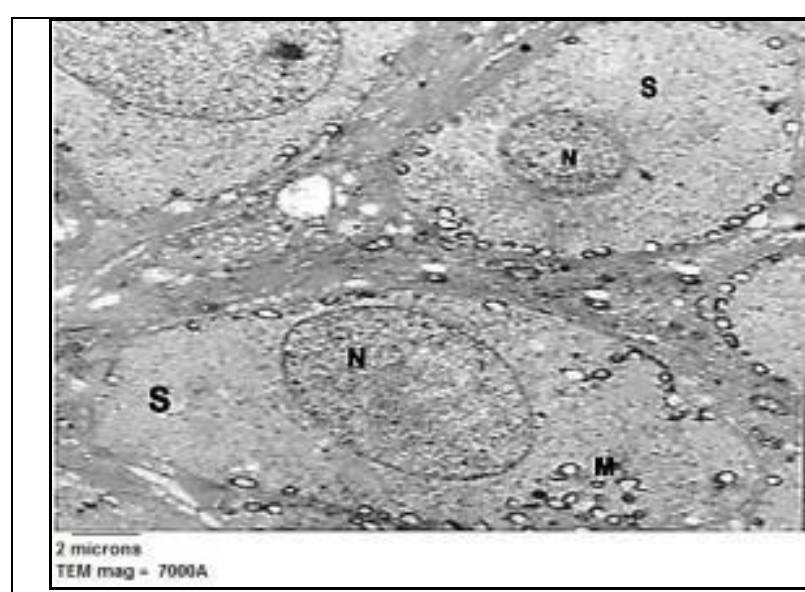

Fig.23: An electron micrograph of an ultrathin section in the testis of the first recovery group (G4) showing spermatids (S) with large ovoid euchromatic nuclei $(\mathrm{N})$. Their cytoplasm contains peripherally located mitochondria $(\mathrm{m})$. (Uranyl acetate\& lead citrate X 7000).

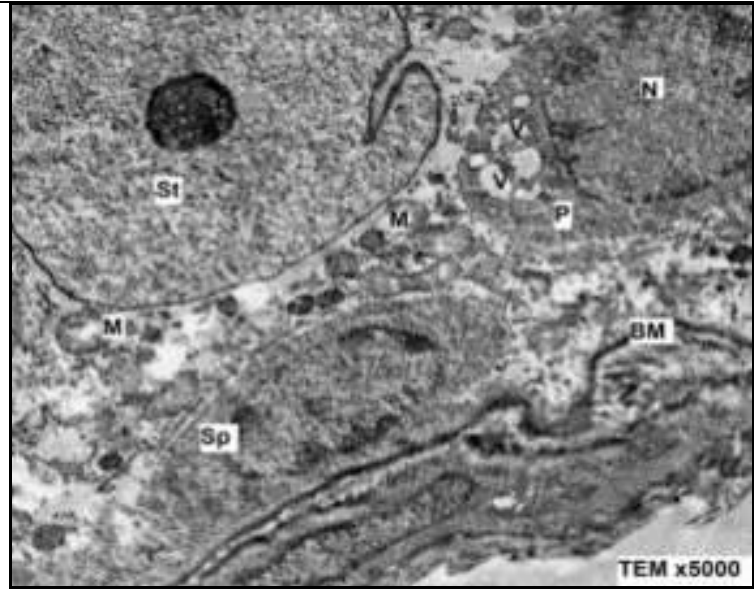

Fig. 24: An electron micrograph of an ultrathin section in the testis of the second recovery group (G5) showing spermatogonia (sp) primary spermatocytes $(\mathrm{P})$ with rounded nuclei $(\mathrm{N})$. Their cytoplasm contains few multiple vacuoles (v). Sertoli cells (st) appear with indented nucleus, prominent nucleolus with multiple mitochondrial vacuoles $(\mathrm{M})$, an irregular thickened basement membrane (BM) (Uranyl acetate\& lead citrate X 5000).

Table (1): Changes in the optical density of collagen casts.

\begin{tabular}{|c|c|c|c|c|c|}
\hline & G.I & G.II & G. III & G. IV & G. V \\
\hline Mean & $\mathbf{7 . 0 5}$ & $\mathbf{7 . 4}$ & $\mathbf{8 . 3}$ & $\mathbf{7 . 2 1}$ & $\mathbf{8 . 1}$ \\
\hline SD \pm & $\mathbf{0 . 0 1}$ & $\mathbf{0 . 0 2}$ & $\mathbf{0 . 0 0}$ & $\mathbf{0 . 0 0 2}$ & $\mathbf{0 . 0 1}$ \\
\hline SE. & $\mathbf{0 . 0 1}$ & $\mathbf{0 . 0 0 0}$ & $\mathbf{0 . 0 0 1}$ & $\mathbf{0 . 0 0 0}$ & $\mathbf{0 . 0 0 0}$ \\
\hline P Value & & $<\mathbf{0 . 0 5}$ & $<\mathbf{0 . 0 5}$ & $<\mathbf{0 . 0 5}$ & $<\mathbf{0 . 0 5}$ \\
\hline Significance & & S. & s. & s. & s. \\
\hline
\end{tabular}

\section{DISCUSSION}

Male infertility is one of the major reproductive disorders that warry the public concern. This disorder has been associated with estrogenic compounds such as endocrine disrupting chemicals (EDCs) ${ }^{(10)}$. The associations between BPA levels and testicular toxicity, semen parameters, and overall male fertility have been extensively studied. Estrogenic activity of BPA has been particularly linked with these injurious effects exerted by BPA. Damaging effects of BPA on the reproductive systems of males and females have been documented in rodents ${ }^{(\mathbf{1 0})}$. A few studies have so far examined this issue for BPA by either toxicological or epidemiological assessments on natal and postnatal reproductive system ${ }^{(\mathbf{1 1})}$.

This present study provided information on BPA toxicity in male albino rats using morphological and statistical approaches.The duration of drug administration in the present work was determined according to the spermatogenic cell cycle duration in rats which was 2 weeks ${ }^{(12)}$, and according to the recommendation of Ayyed et al. (13) who gave Bisphenol A pre- and post natal in male albino rat offsprings. The administered low dose of BPA was 0.3 $\mathrm{mg} / \mathrm{kg}$.B.W. while the high dose was $12 \mathrm{mg} / \mathrm{kg}$ single daily dose. This calculated dose was in agreement with that recommended by Roy et al. ${ }^{(14)}$ and those used by Mourad et al. ${ }^{(15)}$ who studied the effect of Bisphenol A after administering 20, 100 and $200 \mathrm{mg} / \mathrm{kg} /$ day.

Testicular sections obtained from control group (G.1) showed that the seminiferous tubules were lined by Sertoli cells which appeared wedged between the spermatogenic cells with indented basally located nuclei, that possessed prominent nucleolus and exhibits little heterochromatin this was in agreement with, Hess and Vogl (16). Spermatogonia rested on the basal laminae and had small and dark nuclei. Primary spermatocytes in prophase of the first meiotic division, rounded immature spermatids were observed, that was in coincidence with Ibtisham et al. (17). The spermatogonium is a relatively small cell, situated next to the basal lamina of the epithelium, Some of them differentiate into type A dark spermatogonia, with oval nuclei and abundant heterochromatin which gives a dark appearance to the nucleus ${ }^{(\mathbf{1 8})}$. Some spermatids were recorded in the Golgi phase of spermiogenesis and others had acrosomal caps, this was in agreement 
with El-Beltagi et al. ${ }^{(19)}$. Concerning Leydig cells in this group, they are prominent in the interstitum and they are responsible for production of testosterone which is an absolute requirement for the completion of the first wave of spermatogenesis during puberty. In addition, testosterone together with FSH had been found to support spermiation, Katja et al. ${ }^{(20)}$.

In the histopathological studies, the integrity of the epithelium was impaired in some seminiferous tubules, and the primary spermatocytes were separated from basal lamina in some regions in BPA-exposed animals. Spermatogonia, which are immediately adjacent to Sertoli cells in the normal structure basal compartment of seminiferous tubules, were not found in some areas of the seminiferous tubules in BPA groups. In low dose treated group (G.2), sections showed mild affection in most of the S.Ts., some revealed mild vacuolation of spermatogenic cells, Sertoli cells and Leydig cells. Also, S.Ts. showed a thin straight basement membrane, these observations coincide with those of Russell $\boldsymbol{e t}$ al. (21). Also, there were mild disqumation and detachement of germinal epithelium from the basement membrane, the lost germ cells are replaced by intercellular vacuoles, few number of spermatogenic cells are replaced by vacuoles. The spermatogonia showed small pyknotic nuclei. Some of the primary spermatocytes showed shrunken nucleus, other with anirregular nuclear envelope, their cytoplasm showed small intra- and intercellular vacuoles. Also, a diminution in the number of germinal epithelium, the same findings were aggravated in the high dose treated group (G.3), these changes were in agreement with findings reported by Hussein et al. ${ }^{(22)}$. Also, recorded in another study conducted in newborn male rats, BPA was administered subcutaneously $(0.01,0.1$, and $5 \mathrm{mg} / \mathrm{kg}$ $\mathrm{bw})$, and spermatogenesis deteriorated due to BPA exposure ${ }^{(23)}$.

From the previous findings, the seminiferous tubules affected with a marked separation of the spermatogenic cells from the basal laminae could be due to loss of its contact with sertoli cells and as a result of impairment of Sertoli cell function. This in agreement to Hild et al. ${ }^{(24)}$ who said that separation of spermatogenic cells might be due to disruption of smooth endoplasmic reticulum function which affected the interaction between Sertoli and germ cells and resulted in germ cell death. Also, Bashir et al. (25) revealed that the appearance of vacuoles in the seminiferous epithelium was the first notable change, vacuolated and apoptotic spermatogenic cells and might be due to toxic acting on the Leydig cell leading to testosterone deprivation.

In the present study, the primary spermatocytes of rat testis in high dose treated group showed electron dense granules in their nuclei, another one showed degenerative changes such as ruptured nucleus and wide vacuolated spaces. Also, early spermatids showed degenerative changes such as shrunked nuclear membrane (apoptotic nucleus) or damaged nucleus with complete or partial loss of acrosomol cap, their cytoplasm showed patchy areas of vacuolization, also, the interstitial cells of Leydig were shrunken, the nucleus became convoluted and irregular in outline and indented with condensed heterochromatin and prominent nucleolus, the cytoplasm was vacuolated with electron dense bodies of variable size and shape. Those finding were in agreement with Several studies have shown that prenatal BPA exposure can cause various disorders in the rodent testis during adulthood. These conditions were suggested to be impaired spermatogenesis, the presence of immature spermatogenic cells in the testis tubule lumen, the presence of large gaps between Sertoli cells, and tubular lumen expansions ${ }^{(26,27)}$.

The mechanism by which BPA could affect sperm count was explained by Klaunig $\boldsymbol{e t}$ al ${ }^{(28)}$ who concluded that bisphenol A has been shown to accumulate in the fatty tissues and is metabolized by Cytochrome P450. Cytochrome P-450 has been shown to induce ROS that permanently impairs sperm function thereby resulting in decline of sperm counts in men and laboratory animals. Another explanation was reported by Sadek et al. ${ }^{(29)}$ who revealed that BPAinduced impairment of Sertoli cells had been reported by inhibiting endoplasmic reticulum $\mathrm{Ca} 2+$ homeostasisand the ectoplasmic specialization between Sertoli cells and spermatids. BPA may exert both antiandrogenic and estrogenic effects to impair sperm production ${ }^{(30)}$.

Testicular sections obtained from recovery groups (G.4) revealed an increase in the germ cell population, recovery of damaged germinal epithelium, restoration of shape of seminiferous tubules, increase in number of spermatozoa and proper alignment of spermatogonial cells. These results are in accordance with the observations of Khan ${ }^{(31)}$ who have reported that there are higher degree of recovery in germ cells and most of the testicular structure was regenerated, but certain alterations were left which including the vacuolization.

While, testicular sections obtained from the second recovery group (G.5) had still manifested a distorted seminiferous tubules with no return to the normal distribution of epithelial lining, in agreement to Soliman et al. ${ }^{(32)}$ where, the presence of several layers of dark type spermatogonia were observed. Sloughed germ cell were also seen in the lumen. The basement membrane of the seminiferous tubules was irregular and disrupted in some areas. The cells of the stratified germinal epithelium were still containing multiple vacuoles in their cytoplasm.

These observations may be due to that the pubertal period is critical to the influence of toxic dose of Bisphenol regarding testicular androgen secretion, because mitosis occurred in both germ cells and Sertoli 
cells in this age. This in accordance to Hanafy et al. ${ }^{(33)}$.

Masson's trichrome stained sections of the control and first recovery groups, showed mild deposition of collagen fibers in the basement membranes surrounding the tubules and in the interstitial tissues around seminiferous tubules. and in the low dose treated group showed moderate deposition of collagen fibers in in the basement membranes surrounding the tubules and in interstitial tissues around seminiferous tubules. While in the high dose treated \& second recovery groups group showed marked deposition of collagen fibers in the basement membranes which became thickened and wrinkled in the interstitial tissues around the tubules, those finding agreed with Salwa et $\boldsymbol{a l}^{\left({ }^{(34)} \text {. }\right.}$

The collagenous fibers deposition might be dependent on the Sertoli cells basement membrane interactions.Sertoli cells damage and changes in the basement membrane was strictly correlated. The lamina densa adjacent to the altered sertoli cells was usually thickened, infolded and in some portions detached from the sertoli cells plasma membrane. Thickening of basal lamina had been commonly observed in the testes under abnormal conditions and the myoid cells secrete a number of substances including extracellular matrix components fibronectin, type I and IV collagens and proteoglycans ${ }^{(35)}$.

These testicular pathological alterations may explained by the xenoestrogenic properties of BPA that can inhibit testicular growth, as BPA may act as selective toxicant for the male reproductive organs and directly inhibit testicular function as reported by So the xenoestrogen BPA adversely affects the animal reproduction by its action on gonadal steroidogenesis, and subsequently the abnormal release of endogenous steroid hormones.

\section{CONCLUSION}

The results of this study revealed that early-life exposure to Bisphenol A produced testicular toxicity leading to marked serious histological changes of the testis including the spermatogenic cells, spermatids, Sertoli cells and Leydig cells. These testicular changes increased with high dose intake of BPA indicating its cumulative toxic effects that caused hypospermatogenesis, leading to infertility, while its stoppage may hinder those effects. So, these serious complications on testicular structure should be considered when buying any canned foods or drinks, because it is used nowadays on a large scale allover the world .

\section{REFERENCES}

1. Pallotti F, Pelloni M, Gianfrilli D et al. (2020): Mechanisms of testicular disruption from exposure to bisphenol A and phtalates. Journal of Clinical Medicine, 9(2): 471-475.
2. Yirun A, Ozkemahli G, Balci A et al. (2021): Neuroendocrine disruption by bisphenol A and/or di (2ethylhexyl) phthalate after prenatal, early postnatal and lactational exposure. Environmental Science and Pollution Research, 21: 1-14.

3. Barouki R (2017): Endocrine disruptors: revisiting concepts and dogma in toxicology. Comptes Rendus Biologies, 340(9-10): 410-413.

4. Tavares R, Escada-Rebelo S, Correia M et al. (2016): The non-genomic effects of endocrine-disrupting chemicals on mammalian sperm. Reproduction, 151(1): 1-13.

5. Ferguson K, Peterson K, Lee J et al. (2014): Prenatal and peripubertal phthalates and bisphenol $\mathrm{A}$ in relation to sex hormones and puberty in boys. Reprod Toxicol., 47: 70-76.

6. Cariati F, D'Uonno N, Borrillo F et al. (2019): Bisphenol a: an emerging threat to male fertility. Reproductive Biology and Endocrinology, 17(1): 1-8.

7. Srivastava S, Gupta P (2016): Genotoxic and infertility effects of bisphenol A on wistar albino rats. Int J Pharm Sci Rev Res., 41(1): 126-131.

8. Vom S, Hughes $M$ (2005): An extensive new literature concerning low - dose effects of bisphenol-A shows the need for anew risk assessment. Environ Health Perspect., 113: 926-933.

9. European Food Safety Authority (2020): Assessment of new information on Bisphenol S (BPS) submitted in response to the Decision 1 under REACH Regulation (EC) No 1907/2006. European Food Safety Authority (EFSA), 17(4): 1-39.

10.Alabi O, Ologbonjaye K, Sorungbe A et al. (2021): Bisphenol A-induced Alterations in Different Stages of Spermatogenesis and Systemic Toxicity in Albino Mice (Mus musculus). Journal of Health and Pollution, 11(29): 210307-13.

11. Rochester J (2013): Bisphenol A and human health: a review of the literature. Reprod Toxicol., 42:132-155.

12. Conour L, Murray K, Brown M (2006): Preparation of animals for research - issues to consider for rodents and rabbits. ILAR Journal, 47 (4): 283-293.

13. Ayyed H, Abdulameer A, Abdulrazzak N (2013): Effects of Pre-and Postnatal Exposure to Bisphenol- A on the Reproductive Efficacy in Male Albino Rats. J of Kerbala University, 11(3): 158-172.

14. Roy S, Kalita C, Mazumdar M (2011): Histopathlogical effects of Bisphenol A on liver of Heteropneustes Fossilis (Bloch). An International Quarterly Journal of Environmental Sciences the Ecoscan., 1: 187-190.

15. Mourad I, Khadrawy Y (2012): The sensetivity of Liver, Kidney and testis of rats to oxidative stress induced by different doses of Bisphenol A. International Journal of Life Science and Pharma Reviews, 2: 19-28.

16. Hess R, Vogl A (2015): Sertoli cell anatomy and cytoskeleton. In: Griswold M, editor. Sertoli Cell Biology. Elsevier Academic Press; Oxford, Pp. 1-55.

17. Ibtisham F, Wu J, Xiao M et al. (2017): Progress and future prospect of in vitro spermatogenesis. Oncotarget., 8(39): $\quad 66709$ 66727.

18.Pawlina W (2016): Histology of the male reproductive system: Histology Atext Atlas with Correlated Cell and 
Molecular Biology Publisher: Lippincott Williams Wilkins $7^{\text {th }}$ edition, Pp. 352-386.

19.El-Beltagi E, Elwan W, El-Bakry N et al. (2017): Histological and immunohistochemical study on the effect of gibberellic acid on the seminiferous tubules of testis of adult albino rat and the possible protective role of grape seeds proanthocyanidin extract. Tanta Medical Journal, 45(2): 79-84.

20.Katja J, Ilpo T(2015): Morphological and functional maturation of Leydig cells: from rodent models to primates. Human Reproduction Update, 21(3): 310328.

21. Russell L, Ettlin R, Sinha H et al. (1990): Histological and Histopathological Evaluation of the Testis. Clearwater: Cache River Press, 215(2): 365374.

22.Hussein O, Elghait A, Ahmed S (2014): Effect of Nigella sativa oil on aluminum chloride-induced testicular damage in male albino rats: a light and electron microscopic study. Egyptian Journal of Histology, 37(4): 741-755.

23.Xie M, Bu P, Li F et al. (2016): Neonatal bisphenol A exposure induces meiotic arrest and apoptosis of spermatogenic cells. Oncotarget, 7(9): 10606-10612.

24. Hild S, Reel J, Larner J et al. (2001): Disruption of spermatogenesis and Sertoli cell structure and function by the indenopyridine CDB-4022 in rats. Biol Reprod., 65:1771-1779.

25. Bashir N, Shagirtha K, Manoharan V et al. (2019): The molecular and biochemical insight view of grape seed proanthocyanidins in ameliorating cadmiuminduced testes-toxicity in rat model: implication of PI3K/Akt/Nrf-2 signaling. Bioscience Reports, 39: 120.

26. Ma S, Shi W, Wang $X$ et al. (2017): Bisphenol A exposure during pregnancy alters the mortality and levels of reproductive hormones and genes in offspring mice. Biomed Res Int., 2017: 3585809.
27. Kass L, Durando M, Altamirano G et al. (2015): Prenatal Bisphenol a exposure delays the development of the male rat mammary gland. Reprod Toxicol., 54: 37-46.

28. Klaunig J, Wang $\mathrm{Z}$, Pu X et al. (2011): Oxidative stress and oxidative damage in chemical carcinogenesis. Toxicology and Applied Pharmacology, 254(2): 86-99.

29.Sadek K, Abouzed T, Ayoub M (2014): Oxidative and Hormonal Disruptions Underlie Bisphenol AInduced Testicular Toxicity in Male Rabbits. Int J Bio Food Vet Agri Engin, 8(11): 1174-1180.

30.Hatef A, Zare A, Alavi S et al. (2012): Modulations in androgen and estrogen mediating genes and testicular response in male goldfish exposed to bisphenol A. Environmental Toxicology and Chemistry, 31(9): 2069-2077.

31. Khan $R$ (2012): Protective effects of Launaea procumbens on rat testis damage by $\mathrm{CCl} 4$. J Lip Heal Dis., 11: 103-121.

32.Soliman M, Mahmoud B, Kefafy M et al. (2017): Effect of antidepressant drug (fluoxetine) on the testes of adult male albino rats and the possible protective role of omega-3. Menoufia Medical Journal, 30(4): 1135-1142.

33. Hanafy A, Khalil H, Abdel-Rahim W et al. (2016): Testicular functions and sexual behavior in male Japanese quail after exposure to bisphenol A. Asian Journal of Poultry Science, 10(1): 40-51.

34.Salwa S, Seham H, Othman M et al. (2015): Developmental characteristics of rat testicular tissue and the impact of chronic noise stress exposure in the prenatal and postnatal Periods. Anat Physiol J., 35(4): 125-130.

35.Sasso C, Cerri P (2008): morphological evidences indicate that the interference of cimetidine on peritubular component is responsible for detachment and apoptosis of sertoli cells. Rprod. Biol. Endocrinol., 9:18-27. 